Experimental study of the vertical stability of high decay index plasmas in the DIII-D tokamak

This article has been downloaded from IOPscience. Please scroll down to see the full text article.

1990 Nucl. Fusion 302349

(http://iopscience.iop.org/0029-5515/30/11/011)

View the table of contents for this issue, or go to the journal homepage for more

Download details:

IP Address: 128.178.125.142

The article was downloaded on 18/02/2013 at 08:56

Please note that terms and conditions apply. 


\title{
EXPERIMENTAL STUDY OF THE VERTICAL STABILITY OF HIGH DECAY INDEX PLASMAS IN THE DIII-D TOKAMAK
}

\author{
J.B. LISTER*, E.A. LAZARUS**, A.G. KELLMAN, J.-M. MORET*, \\ J.R. FERRON, F.J. HELTON, L.L. LAO, J.A. LEUER, \\ E.J. STRAIT, T.S. TAYLOR, A.D. TURNBULL \\ General Atomics, \\ San Diego, California, \\ United States of America
}

\begin{abstract}
Experiments on the stabilization of highly elongated, vertically unstable plasmas were carried out on the DIII-D tokamak. Identification of the closed-loop transfer function showed that vertical stability could be usefully modelled as a second order dynamical system. The effect of varying the controller gains and the vertical field decay index was studied and found to be qualitatively as predicted by a low order model proposed previously. The implementation of a new hybrid inboard/outboard coil positional control with differing controller dynamics allowed operation of DIII-D up to $92 \%$ of the limiting equilibrium field decay index of the vacuum vessel for the plasmas used. This improved control allowed operation at a plasma elongation $x$ of up to 2.5 .
\end{abstract}

\section{INTRODUCTION}

One method currently under study for increasing the viability of the tokamak as a fusion energy device is to operate with more strongly non-circular plasma crosssections. In this way the current carrying capability can be significantly enhanced, leading hopefully to higher attainable values of the plasma beta, which scales theoretically and experimentally as $\beta \approx \mathrm{I}_{\mathrm{p}} / \mathrm{aB}_{\mathrm{t}}$ $\approx\left(1+\kappa^{2}\right) / 2 \mathrm{Aq}$, where $\mathrm{I}_{\mathrm{p}}$ is the plasma current, $\mathrm{B}_{\mathrm{i}}$ is the toroidal field, $a$ is the horizontal minor radius, $\kappa$ is the plasma elongation, $A$ is the aspect ratio and $q$ is the safety factor. Furthermore, higher elongation allows a higher value of $I_{p} / B_{t}$ at fixed $q$, leading to better energy confinement.

To achieve the desired elongations, a strong quadrupole field must be added to the radially stabilizing vertical field. The relative strengths of the quadrupole and vertical (dipole) fields determine the curvature of the field lines, which is conveniently expressed as the equilibrium field decay index

$n \equiv-\left.\frac{X}{B_{z}} \frac{\partial B_{z}}{\partial X}\right|_{X=x_{0}}$

* Centre de recherches en physique des plasmas, Association Euratom-Confédération Suisse, Ecole polytechnique fédérale de Lausanne, 21, avenue des Bains, $\mathrm{CH}-1007$ Lausanne, Switzerland.

** Fusion Energy Division, Oak Ridge National Laboratory, Oak Ridge, TN 37831, USA. and which must be negative for $\kappa>1 . \mathrm{X}$ is the plasma major radial co-ordinate, $X_{0}$ is the plasma major radius, the symbol $R$ being reserved to denote electrical resistance. The toroidal plasma current in such a field configuration is positionally unstable in the vertical direction, and we must dynamically keep it close to a given metastable position using active feedback control of the radial field. All tokamaks with an elongation greater than that obtained naturally in a purely vertical field $(n=0)$ have had to provide positional stabilization of this naturally unstable system.

The analysis and understanding of the results of this paper depend on the formalism and discussions developed in Ref. [1]. This introduction repeats the salient points of the earlier study. In Ref. [1] we showed that the limiting value for the decay index is related to the critical index of the shell,

$\mathrm{n}_{\mathrm{c}} \equiv \frac{2 \mathrm{M}_{\mathrm{vp}}^{\prime 2} \mathrm{X}_{0}}{\mu_{0} \Gamma \mathrm{L}_{\mathrm{v}}}$

$\Gamma \equiv \frac{L_{\text {ext }}}{\mu_{0} \mathrm{X}_{0}}+\frac{\ell_{\mathrm{i}}}{2}+\beta_{\mathrm{p}}+\frac{1}{2}$

Note that $n_{c}$ is defined as a positive quantity, and that plasmas experience vertical instability for negative values of $\mathbf{n}$. The ideal limit for rigid-body vertical stability is $n / n_{c}>-1$. Throughout the paper, the subscripts p, $v$ and a refer to plasma, vessel (or shell) and 
active feedback coil, respectively. $L$ is the symbol for self-inductance and $\mathrm{M}$ is mutual inductance. $\mathrm{I}$ and $\mathrm{V}$ are current and voltage. The prime denotes partial differentiation with respect to vertical position. The notation otherwise follows that of Ref. [1].

Two problems will arise in the future. Firstly, the size and current of the plasmas to be stabilized are increasing, leading to significantly greater power handling requirements. Secondly, in experiments such as the DIII-D tokamak [2] and the TCV tokamak [3] we must stabilize more elongated plasmas, moving to more intrinsically unstable configurations. For both of these cases it is important to develop a reliable operational understanding of the vertical stabilization problem, upon which an optimal control strategy can be based, allowing a minimization of the power requirements. The increase in the forces on the vessel and the poloidal coil system resulting from the motion of a high current plasma with greater elongation presents a further motivation. A final motivation is given by the fact that the poloidal system of a large tokamak represents a significant part of the system cost. The design of this system must, therefore, be carefully optimized.

In Ref. [1], an approach has been evolved for modelling and improving the DIII-D tokamak vertical position feedback control. The starting point for the vertical stability dynamics is a rigid massless plasma. The plasma is characterized by its size, current, poloidal beta, and the internal and external inductances. These are incorporated so as to be consistent with radial force balance, assuming stationary fields.

In the inhomogeneous equilibrium field, the plasma current experiences a vertical force given by

$F_{z}=\int_{\text {plasma volume }} j_{\phi}(\vec{r}) B_{r}(\vec{r}) d^{3} \vec{r}$

To retain the dominant physics while remaining algebraically simple, this force was approximated as $\mathrm{F}_{\mathrm{z}} \approx 2 \pi \mathrm{X}_{0} \mathrm{I}_{\mathrm{p}} \mathrm{B}_{\mathrm{r}}\left(\mathrm{X}_{0}\right)$. The vacuum vessel provides a restoring force via the radial field resulting from the image currents induced by the plasma motion. The poloidal distribution of the vessel current is usefully decomposed into orthogonal modes (Ref. [1],

Appendix A). The first antisymmetric mode dominates the vertical control owing both to its long decay constant and to its large radial field on axis. Retaining only the dominant vessel modes important for plasma control again simplifies the algebra, while retaining the physics that is important for plasma control. Up-down symmetric pairs of active feedback coils fed in antiparallel were added to the model in terms of their mutual inductance to the vessel current modes and their radial field at the plasma axis.

This simplified model leads to three determining equations. The first equation

$$
\frac{\mu_{0} I_{p} \Gamma}{2 X_{0}} n z-\frac{d M_{v p}}{d z} I_{v}-\frac{d M_{a p}}{d z} I_{a}=0
$$

is the equation for vertical force balance for a massless plasma; the plasma is located at the position where the radial field vanishes.

We add the two circuit equations for the first antisymmetric vessel eigenmode current $I_{v}$ and the active antisymmetric coil current $I_{a}$

$$
\begin{aligned}
& L_{v} \frac{d I_{v}}{d t}+R_{v} I_{v}+M_{a v} \frac{d I_{a}}{d t}+\frac{d M_{v p}}{d z} \frac{d z}{d t} I_{p}=0 \\
& L_{a} \frac{d I_{a}}{d t}+R_{a} I_{a}+M_{a v} \frac{d I_{v}}{d t}+\frac{d M_{a p}}{d z} \frac{d z}{d t} I_{p}=V_{a}
\end{aligned}
$$

The applied active voltage in Eq. (6) is defined by a proportional-derivative (PD) controller,

$$
\frac{V_{a}(t)}{I_{p} L_{a}}=G_{z}\left[z(t)-z_{r e f}(t)\right]+G_{v} \frac{d}{d t}\left[z(t)-z_{r e f}(t)\right]
$$

where $G_{z}$ and $G_{v}$ are the positional proportional and derivative gains, respectively, and $I_{p}$ is assumed constant. In what follows, the values of $G_{z}(m \cdot s)^{-1}$ and $G_{v}(m)^{-1}$ will be given in MKSA units unless explicitly noted otherwise.

These three determining equations lead to a characteristic equation corresponding to a dynamical system model of only second order. One aim of this paper is to determine whether such a simple dynamical system correctly represents the experimentally observed vertical movement of an elongated plasma.

The model as derived in Ref. [1] did not explicitly incorporate poloidal shaping coils other than those fed with the vertical control signal. This point is discussed later in the paper (Section 3.3).

The influence of different PD gain settings and different vertical field decay indices was studied systematically in Ref. [1]. It is characteristic of such a 


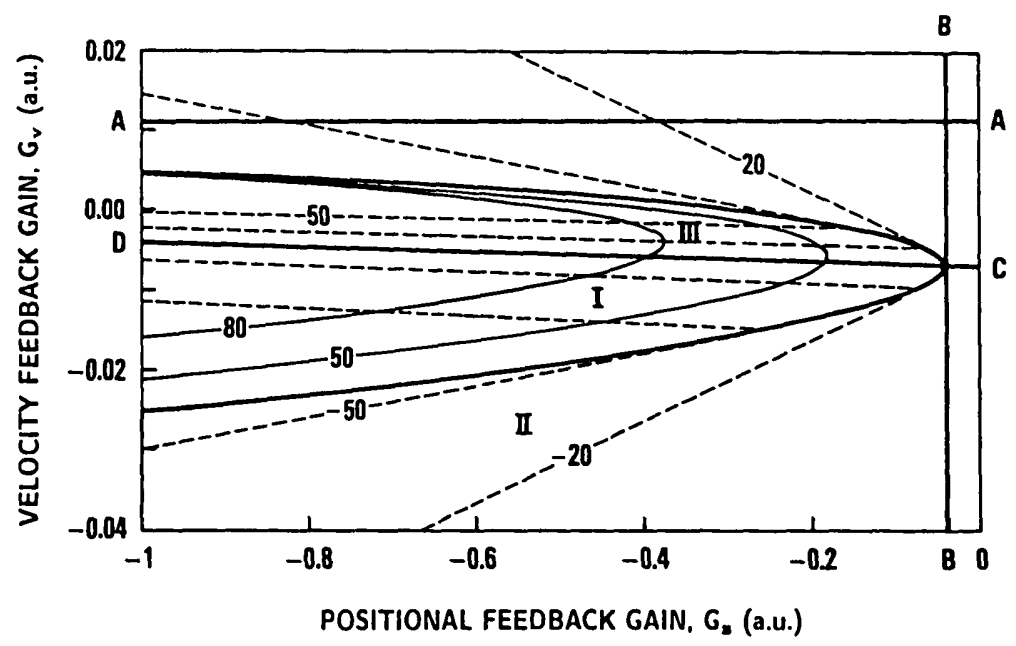

FIG. 1. Schematic of the operating regions in the $G_{z} G_{\mathrm{v}}$ plane using the $F 2$ coils. The stable operating region is below $A-A$, below $C-D$, and to the left of $B-B$. The thick curve separating Regions $I$ and II is the $\omega=0$ contour. The dashed lines are contours of constant $\gamma$ (in $s^{-1}$ ) and the thin solid lines are contours of constant $\omega(\mathrm{rad} / \mathrm{s})$. In Region I the solutions are stable and underdamped, in Region II they are overdamped, and in Region III the solutions are unstable and oscillatory.

second-order system that, once the system parameters are determined, the system stability can be characterized in terms of the derivative and the proportional gains of the controller. The general behaviour of the plasma position control is reproduced in Fig. 1. The axes represent the proportional and derivative gains, Eq. (7). The stable operating regime is below the sloping line C-D and to the left of the vertical line B-B, i.e. Regions I and II. Within this stable region, the response can be oscillatory (Region I) or overdamped (Region II). The solid contours give the oscillation frequency $(\omega)$ and the dashed lines give the real growth rate $(\gamma)$, which are negative in the stable region. This diagram is characteristic of any secondorder system which is stabilized using a PD controller. It is determined for a particular value of $n$. As $n$ increases, the line $C-D$ moves downwards. Increases in $-G_{v}$ increase the damping and therefore the sluggishness of the system, whereas increases in $-G_{z}$ decrease the damping. The operating point must be chosen to be consistent with series of such stability diagrams which cover the range of $n / n_{c}$ over the desired tokamak operating range ( 0 to -1 in our case). We shall refer repeatedly to this diagram in describing the experimental results.

Several important features of the system were identified in Ref. [1]:

- There is a critical decay index, $\mathbf{n}_{c}$, above which control is not possible. Beyond this limit, the plasma will in fact move with the Alfven velocity. The vessel current restoring force is inadequate to stabilize the plasma.

- The vessel image current induced by a vertical movement is predominantly on the outboard side owing to toroidicity.

- For $\mathbf{n}>-\mathrm{n}_{\mathrm{c}}$, the plasma can be stabilized, provided a suitable choice of the active coil is made. Since the vessel shields the field from the outboard active coils very effectively, inboard coils are needed to reach the ideal limit.

- A vertical field decay index $n_{a}$ is associated with the active coils; above this index, active feedback stabilization requires velocity gain.

- The power requirements are sensitive to the coil placement. The voltage requirement scales as $\mathrm{e}^{\left(-7 n / n_{c}\right)}$, and the bandwidth requirement is the openloop growth rate of the mode (Ref. [1], Section 4).

One result of this study is that there is no single choice of a control coil pair which provides both the stability to reach the ideal limit and the uniformity of the radial field desired for adjustment of the equilibrium vertical position. The suitability of a particular coil set for providing vertical stability is determined primarily by the strength of its interaction with the plasma relative to the strength of its interaction with the vacuum vessel. As an alternative to a poor choice of a single coil pair, a multiple time-scale controller using two coil pairs was developed. In this new hybrid control scheme, the outboard coils, which produce a large and 
uniform radial field over most of the plasma volume, are driven only on the vessel $L / R$ time-scale. In this way, they do not induce large vessel currents which destabilize the plasma. Control on a time-scale faster than the instability growth time is provided by the inboard coils. The radial field produced by these currents is able to penetrate the vessel rapidly $\left(\tau \approx 0.16 \mathrm{~L}_{v} / R_{v}\right.$ ), since they interact primarily with the higher order (spatial frequency) terms in the poloidal distribution of vessel current. $L_{v} / R_{v}$ is the time constant of the slowest, antisymmetric vessel current eigenmode (Eq. (5)); the higher order modes decay much faster. On the other hand, these inboard coils are less attractive for providing the equilibrium radial field establishing the plasma position on the longer time-scale, since they require a far greater coil current for a given radial field. On DIII-D, this hybrid control has allowed the control of plasmas at decay indices close to $-n_{c}$, the ideal MHD positional stability limit.

The motivation of the present paper is therefore fourfold: (1) We report on a series of experiments which demonstrate that the behaviour of the vertical control system is dominantly second order. (2) By examining a variety of plasmas with systematic variations in the decay index, as well as the derivative and proportional gains, we demonstrate the validity of the model developed in Ref. [1]. (3) The improvement in vertical control using the hybrid system previously discussed is substantiated. (4) We report results using this hybrid control to extend the range of elongation up to $k=2.5$.

The paper is organized as follows. In Section 2 we present the details of the experimental set-up. In Section 3 we analyse the closed-loop response to a step perturbation and extract the order of the transfer function. In Section 4 we examine the effects of variations in decay index and controller gains and compare the experimental results with the model predictions of Ref. [1]. In Section 5 we discuss the improvements observed with the hybrid control system and operation very close to the ideal stability limit. In Section 6 we report on higher elongations that we have achieved, in particular plasmas with $\kappa=2.5$. Section 7 summarizes the work.

\section{EXPERIMENTAL CONDITIONS}

The experiments described were carried out on the DIII-D tokamak [2], with nominal parameters $\mathrm{X}_{0}=1.67 \mathrm{~m}, \mathrm{a}=0.67 \mathrm{~m}, \mathrm{~B}_{\phi}=2 \mathrm{~T}, \mathrm{I}_{\mathrm{p}}=1 \mathrm{MA}$.

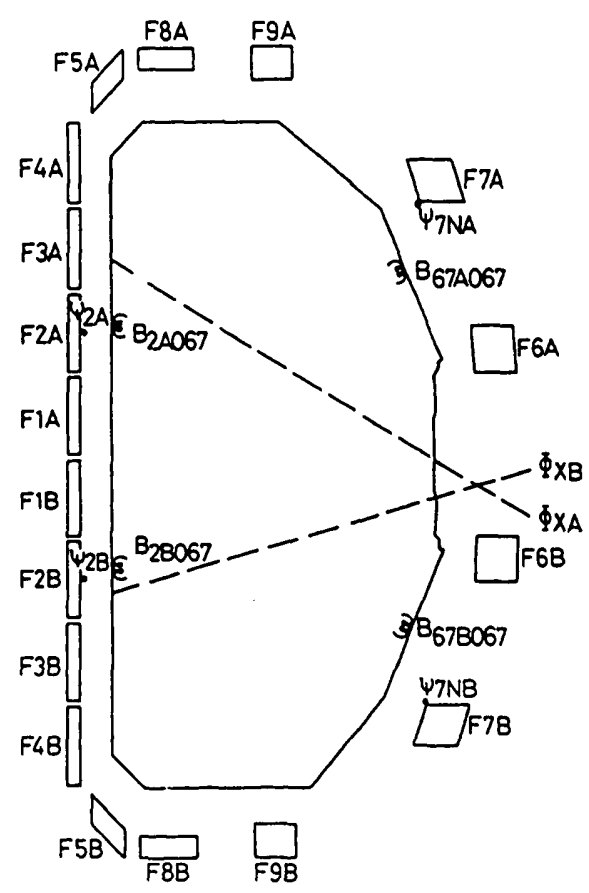

FIG. 2. Layout of the DIII-D tokamak, showing the poloidal field coils, the effective limiter outline, the vertical position detection coils, and the soft $X$-ray chords used to monitor the vertical position.

The poloidal field system is extremely flexible, with 18 close-fitting, independently controllable coils, labelled FnA and FnB in Fig. 2. The equilibrium field is programmed using all the coils, and the vertical position control is superimposed onto selected coil pairs. The vertical position is detected using a combination of flux loop signals $(\psi)$ and poloidal field pickup coils (B), shown in the figure, such that

$$
\begin{gathered}
I_{p} \cdot z=C_{1}\left(\psi_{7 N A}-\psi_{7 N \mathrm{NB}}\right)+C_{2}\left(\psi_{2 A}-\psi_{2 B}\right) \\
+C_{3}\left(B_{67 \mathrm{~A}}-B_{678}\right)+C_{4}\left(B_{2 A}-B_{2 B}\right)
\end{gathered}
$$

where $\mathrm{z}$ is the vertical displacement with respect to the vessel centre. The subscripts correspond to the different poloidal locations, as shown in Fig. 2. This representation of the vertical position in terms of the available magnetic signals has been shown to be accurate for quiescent plasmas using a regression analysis of a large equilibrium database from which the coefficients $C_{i}$ were determined. With regard to the equilibrium aspect of vertical control, the flux loops alone are adequate to define $z$. However, the control of an unstable equilibrium requires the use of field measurements inside the vessel. Because of flux conservation of the plasma-vessel system when the plasma undergoes a 
(a)

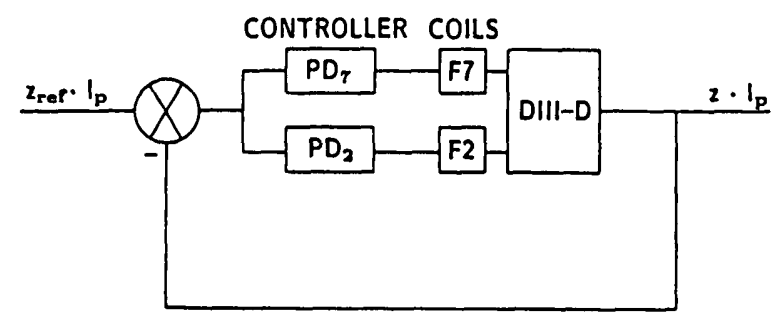

(b)

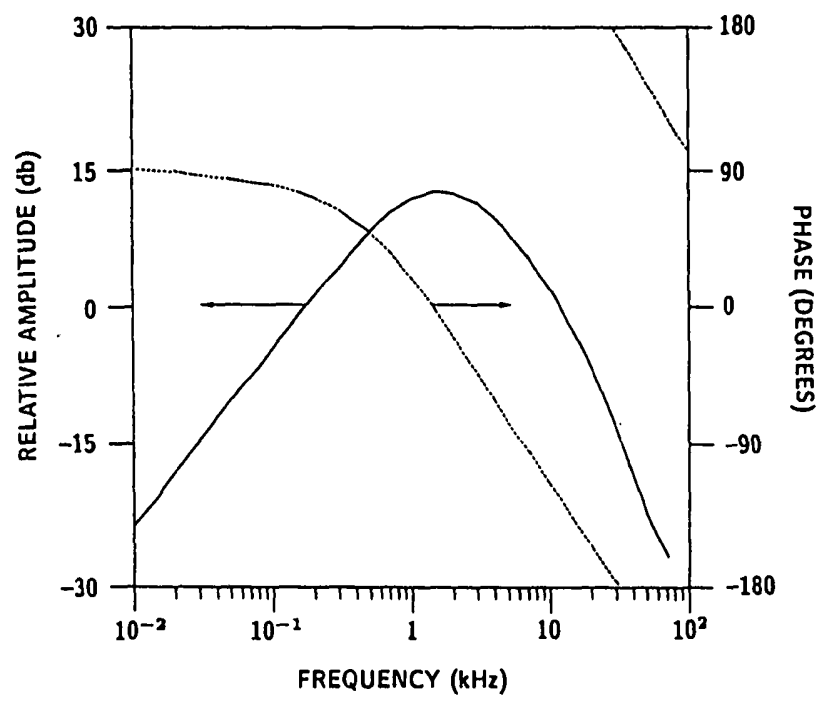

(c)

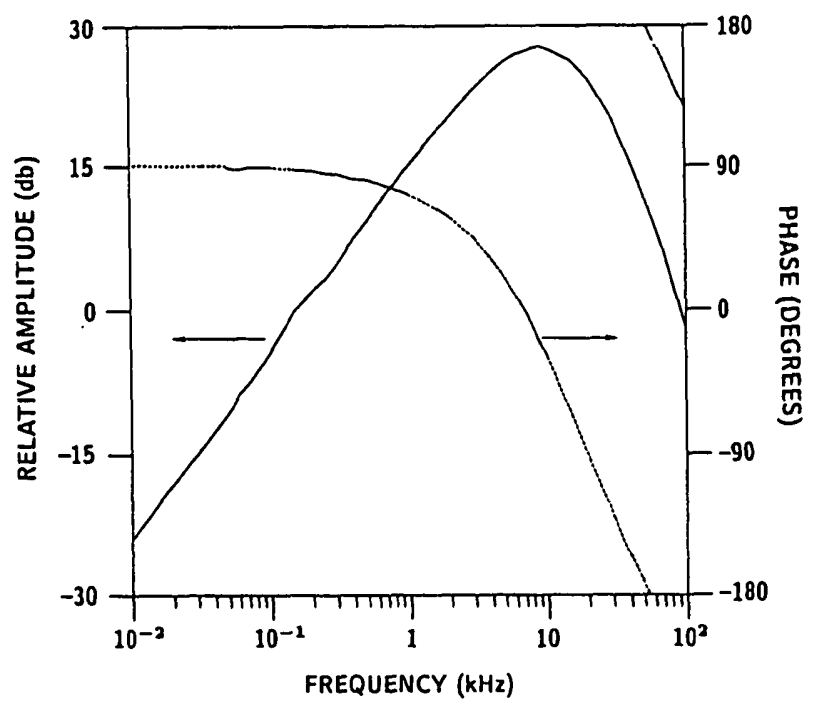

FIG. 3. (a) Schematic of the hybrid controller. (b) Amplitude and phase of the derivative term for the F7 controller. (c) Amplitude and phase of the derivative term for the F2 controller. position excursion, the flux loop signal will be delayed, whereas the internally mounted poloidal field pickup coils react instantaneously. Without the internal probes, the phase shift in the $\mathrm{z}$ measurement is not acceptable once the decay index is less than $-n_{a}$.

Since it was unclear whether or not the stability of the control loop using this position estimate would still be susceptible to problems due to the image currents in the vessel or the controlling currents in the active coils (Ref. [1], Section 7), a second uncalibrated measurement was also made, using the soft X-ray emission from the plasma. We defined the soft $X$-ray vertical asymmetry factor $z_{\mathrm{X}}$ to be

$\mathrm{z}_{\mathrm{X}}-\frac{\Phi_{\mathrm{XA}}-\Phi_{\mathrm{XB}}}{\Phi_{\mathrm{XA}}+\Phi_{\mathrm{XB}}}$

where $\Phi_{\mathrm{XA}}$ and $\Phi_{\mathrm{XB}}$ are the emission signals from upper and lower soft X-ray chords, shown in Fig. 2. These chords were chosen to be on the steep part of the soft X-ray radial profile so as to provide the best sensitivity to a vertical displacement, and a minimum sawtoothing signal. The available signals in fact led to a choice of slightly up-down asymmetric chords. The magnetically derived z-position always agreed well with $\mathrm{z}_{\mathrm{X}}$, at least down to a $1 \mathrm{~ms}$ time-scale.

The vertical feedback control was performed using two PD controllers with the magnetically derived vertical position, $I_{p} \cdot z$, as the input. The $z$-position error signal was applied either to the outboard F7 coil pair or to both the F7 coils and the inboard F2 coils by different controllers (Fig. 3(a)). The use of different controller dynamics for the generation of the inboard and the outboard radial field is an essential part of the hybrid control of the vertical position. Simply applying the same corrector signal to many poloidal coils would not lead to the same improvement. In all cases, we refer to the antisymmetric component of the command signal to the FnA and FnB coils as the demand signal of the Fn coils, treating their currents in a similar way. No attempt was made to incorporate an integral term into the controller, since the problems of precision were not the aim of these experiments.

Figures 3(b) and 3(c) show the curves of the amplitude and phase of the two derivative terms in the controllers, as measured with a network analyser. The F7 controller was constructed to roll off at approximately $2 \mathrm{kHz}$, since these outboard coils have a negligible effect at this frequency because of the shielding by the dominant antisymmetric mode $\left(L_{v} / R_{v} \approx 5 \mathrm{~ms}\right)$ of the vacuum vessel. It should be 


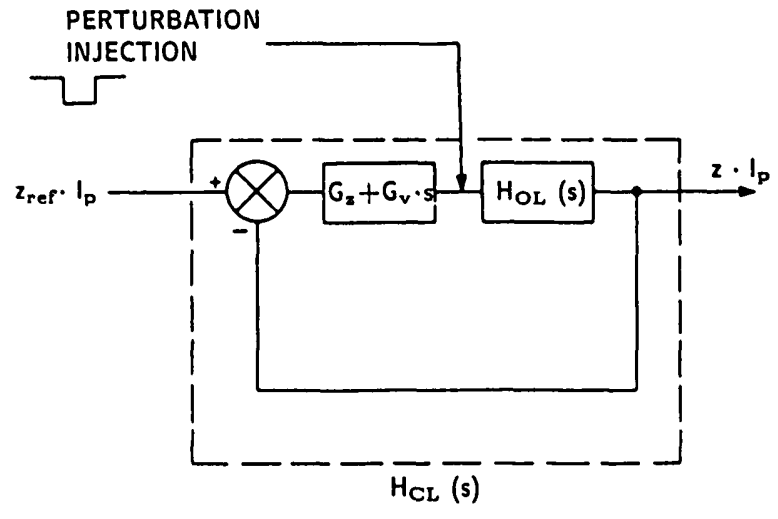

FIG. 4. Schematic of the vertical position control loop, shown isolated from the rest of the poloidal system for simplicity.

noted that the phase shifts for these controllers become significant at considerably lower frequencies than those at $3 \mathrm{db}$. In such a controller system, noise is a problem. We cannot limit the bandwidth to that which is of interest here, because of the resultant phase shifts. At the same time we want to avoid driving the power supplies with this high frequency noise which can easily lead to saturation. This effectively limits the level of derivative gain permissible in the circuit. For our system, the 'noise' will be a combination of plasma noise and electrical circuit noise, all MHD activity other than $n=0$ contributing noise in this sense. The power supplies on the F7 and F2 coils are chopper amplifiers with chopping frequencies of typically $2 \mathrm{kHz}$ [4].

To understand the dynamical system which we must stabilize, we performed a set of perturbation injection experiments. The perturbation chosen was a squarewave modulation $(5 \mathrm{~Hz})$ introduced into the control loop (Fig. 4). The resulting vertical step was of the order of $1-2 \mathrm{~cm}, 1 \%$ of the plasma height. In this figure, $\mathrm{H}_{\mathrm{OL}}(\mathrm{s})$ is the unstable open-loop transfer function between the command signal defined in Eq. (7) and the vertical plasma position. This transfer function includes the pair of power supplies fed in antiparallel, to provide the radial field, the reaction of the vessel currents and the plasma motion, plus all the other active circuits in DIII-D. The active power supplies, fed in antiparallel for the vertical control, are also used individually for the various plasma shaping control loops (these are not shown, for simplicity). If we consider $\mathrm{H}_{\mathrm{OL}}(\mathrm{s})$ together with the feedback loop as a closed-loop system $\mathrm{H}_{\mathrm{CL}}(\mathrm{s})$, shown by the dotted line in Fig. 4, then we can study the effect of the feedback gains on the closed-loop stability.
If we perturb the reference input (Fig. 4), then the transfer function between the injected signal and the plasma position is the closed-loop transfer function $\mathrm{H}_{\mathrm{CL}}(\mathrm{s})$. In practice, two problems arise with this technique. Firstly, the derivative action of the controller, even if mildly filtered, leads to a large spike on the control amplifier demand signal and to saturation of the loop to be analysed. Secondly, the duration and form of the resultant vertical motion are unfavourable for an accurate measurement of the poles. If we inject a perturbation elsewhere in the closed loop, specifically just after the controller (Fig. 4), the closed-loop poles are unchanged. Only the roots of the transfer function are modified: the DC gain becomes dependent on $G_{z}$, and the phase depends on the ratio $G_{v} / G_{z}$. It was found that the signals obtained with the latter technique could be easily analysed, and this technique was used in the experiments discussed in the following sections.

\section{EXPERIMENTAL DETERMINATION OF THE SYSTEM STRUCTURE}

To optimize the controller for the vertical control of DIII-D, it was essential to determine the dynamics of the vertical movement. This section shows that a second-order dynamical model reproduced the experimentally observed plasma motion satisfactorily, and that this was a good model on which the control improvements were to be based.

\subsection{Qualitative behaviour}

Before attempting to identify $\mathrm{H}_{\mathrm{CL}}(\mathrm{s})$ formally, we inspect the sytem response for three characteristic discharges using the previous DIII-D vertical control on the F6 and F7 coils in parallel, with the drive signal of the F6 coils much smaller than that of the F7 coils. Figure 5(a) shows a discharge in which the step response was overdamped, with a rise time of approximately $20 \mathrm{~ms}$. The controller had both a large value of $G_{v}$ and a large value of $G_{z}$. Figure 5(b) shows an oscillating but damped response, which is therefore still stable, with negligible velocity gain $G_{v}$. Finally, we obtained an oscillatory but unstable discharge (Fig. 5(c)), with gains similar to those in Fig. 5(b), but at a slightly larger decay index. In this case the positive pole, which defines the envelope of the unstable growth, remained small, around $10 \mathrm{~s}^{-1}$, being vertically unstable in the closed-loop control sense rather than in the ideal MHD sense. In this unstable case there is clearly no square wave excitation neces- 


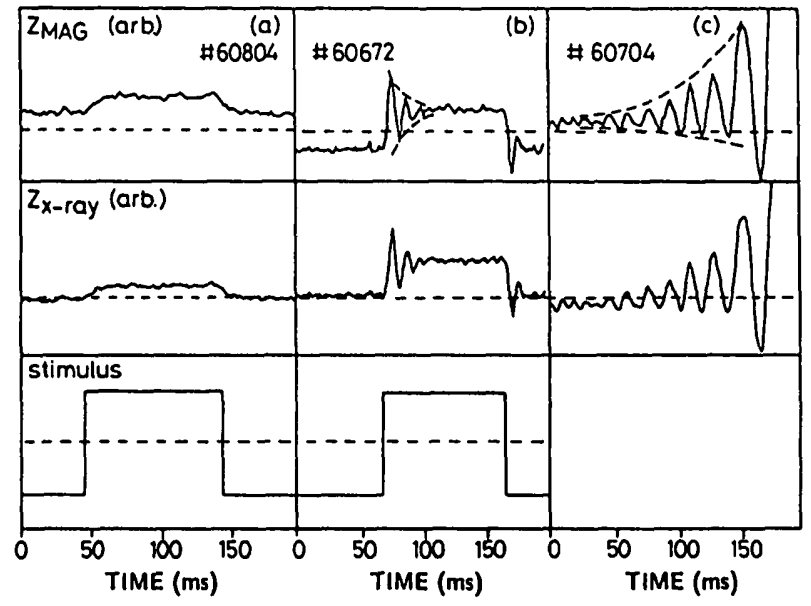

FIG. 5. Characteristic system response of the vertical position: (a) overdamped, (b) oscillatory but stable, and (c) oscillatory and unstable. The vertical position is indicated by both the magnetic and the soft $X$-ray asymmetry measurements.

sary to stimulate the system. In all these cases, there is no evidence of any difference between the response of the magnetic measurements and that of the soft X-ray measurements of the vertical position. These responses are characteristic of a second-order system.

Two points concerning the system linearity have to be discussed. Firstly, when larger amplitude square waves were injected in conditions which lead to an oscillatory response, the oscillation frequency slowed down as the amplitude increased. The power supplies were being progressively saturated as the amplitude increased. This result can be interpreted as being equivalent to a progressive reduction of the controller proportional gain, shown to reduce the oscillation frequency (Fig. 1). Care was taken to keep the power supplies unsaturated for the quantitative response identification. Secondly, the chopper amplifier transfer function is itself non-linear. The two coil supplies may have different net currents for a given plasma boundary shape, resulting in differing bandwidths for the upper and lower coil responses. This asymmetry, which renders the vertical position modulation asymmetric, is visible in Fig. 5(b), where the leading and trailing edge responses are noticeably different. In what follows, the chopper transfer function is assumed to be linear.

\subsection{System identification}

Having qualitatively inspected the closed-loop response, we can proceed with a formal identification of the dynamical system. The system identification procedure can be envisaged in Fig. 6. The goal is to estimate the modelled transfer function $\mathrm{H}^{*}(\mathrm{~s})$ which, when stimulated by the given experimental input $x(t)$, in our case the square-wave modulation, outputs a waveform $y(t)$, which is as similar as possible to the measured experimental waveform $y(t)$. A system identification tool based on this diagram [5] had already been developed for dynamical studies on TCA [6]. The transfer function of the model is of the form

$$
H^{*}(s)=g \frac{\prod_{p=1}^{p}\left(1-\frac{s}{s_{0 p}}\right)}{\prod_{q=1}^{Q}\left(1-\frac{s}{s_{00 q}}\right)}
$$

Such a form represents any physical system described by ordinary differential equations. The DC gain $\mathrm{g}$ and the set of zeros $\left(\mathrm{s}_{01} \ldots \mathrm{s}_{0 \mathrm{p}}\right)$ and poles $\left(\mathrm{s}_{001} \ldots \mathrm{s}_{00 \mathrm{q}}\right)$ completely parametrize such a system. The set of equations (4) through (7) yielded a quadratic denominator $(Q=2)$ and numerator $(P=2)$.

The available experimental data are not in the continuous time variable, but are sampled and stored digitally. Rather than continuous variables $x(t)$ and $y(t)$, we have discretized data samples $x\left(t_{i}\right)$ and $y\left(t_{i}\right)$. In order to use these sampled data points, we apply the bilinear transformation given by

$s-\frac{2}{\Delta \mathrm{T}}\left(\frac{1-\mathrm{z}^{-1}}{1+\mathrm{z}^{-1}}\right)$

where $\Delta \mathrm{T}$ is the sampling period and $\mathrm{z}^{-1}$ is the unit delay operator, such that $z^{-1} \cdot y\left(t_{i}\right)=y\left(t_{i-1}\right)$.

Applied to the transfer function defined in Eq. (10), this transformation produces a transfer function expressed as a rational function in $\mathrm{z}^{-1}$ whose numerator and denominator are $\mathrm{B}^{\prime}\left(\mathrm{z}^{-1}\right)$ and $\mathrm{A}^{\prime}\left(\mathrm{z}^{-1}\right)$, respectively:

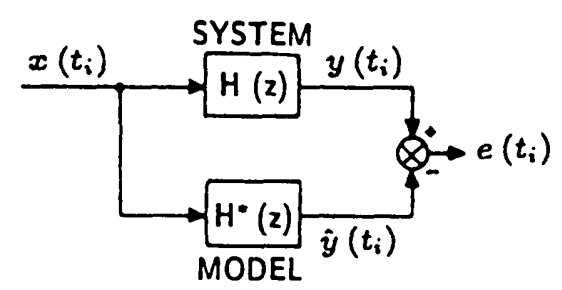

FIG. 6. Schematic of the equivalent system identification problem. $x\left(t_{i}\right)$ and $y\left(t_{i}\right)$ are the input and output discrete-time data;

$\hat{y}\left(t_{i}\right)$ is the modelled output data. 


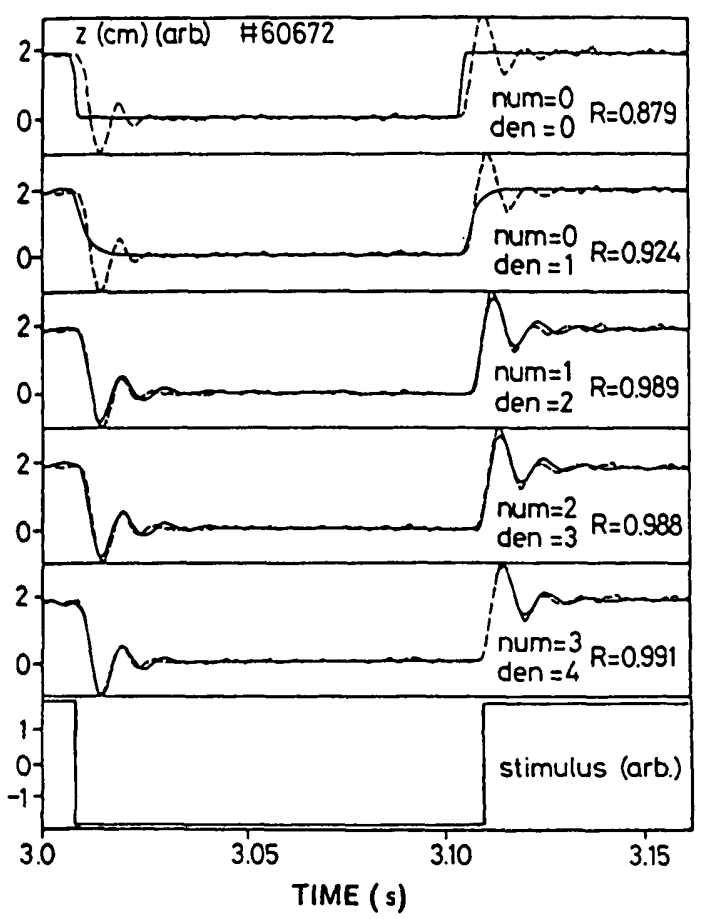

FIG. 7. Results from the system identification code for the oscillatory but stable case of Fig. $5(b)$. The denominator is tested up to fifth order. The measure fitted correlation coefficient $R$ is given.

During the fitting procedure we evaluate the z-plane transfer function

$H^{*}\left(\mathbf{z}^{-1}\right)=\frac{\mathbf{B}^{\prime}\left(\mathbf{z}^{-1}\right)}{\mathbf{A}^{\prime}\left(\mathbf{z}^{-1}\right)}$

Recursive use of the delaying property of the $\mathrm{z}^{-1}$ operator directly allows us to estimate the fitted output $\hat{y}\left(t_{i}\right)$ on the basis of the excitation signal $x\left(t_{i}\right)$. The coefficients of the two polynomials $\mathrm{A}^{\prime}$ and $\mathrm{B}^{\prime}$ are chosen so as to minimize the mean square error between the actual output $y\left(t_{i}\right)$ and the estimated output $\hat{y}\left(t_{i}\right)$ [7]. The $z \rightarrow s$ inverse mapping then allows us to reconstruct the s-plane transfer function $H(s)$ within the frequency band $f<(2 \Delta T)^{-1}$, given by the Shannon limit.

The experimental sampling interval of $1 \mathrm{~ms}$ therefore gives us an estimate of the transfer function $\mathrm{H}^{*}(\mathrm{~s})$ up to a frequency of $500 \mathrm{~Hz}$. As we approach this limit, however, the accuracy of the modelled transfer function will degrade. On the other hand, for frequency components very much slower than the Shannon limit, we become oversampled, and the data must be pretreated, for example by a decimating filter [7].
The numerator of $\mathrm{H}(\mathrm{s})$ must be at least one order smaller than the denominator if the determining equations of the system are physically meaningful. We have allowed the numerator to have this maximum order when performing the modelling for different denominator orders.

This fitting procedure was carried out on the data of Fig. 5(b), box-car averaging three square-wave cycles to improve the signal-to-noise ratio. The results in Fig. 7 show the measured response (dashed curve) and the modelled response (solid curve) to the square wave stimulus. The agreement improves significantly as the order of the denominator increases to second order, after which the general form of the fitted response is only slightly modified by the presence of more degrees of freedom. This saturation is quantitatively shown in Fig. 8, where the correlation coefficient, given by

$R=1-\frac{\left\langle(y-\hat{y})^{2}\right\rangle}{\left\langle(y-\langle y\rangle)^{2}\right\rangle}$

increases up to a second-order denominator. The pronounced knee (Fig. 8) in this correlation coefficient when the model order is equal to, and then exceeds, the dominant order of the identified system is frequently used as a criterion to choose the optimum model structure [7].

The poles of the transfer function are common to all transfer functions of a given physical system. They therefore contain all the information on the dynamics of the system itself. The numerator of the transfer function is related to the observation of an action on the system, and depends on the choice of inputs and outputs, in our case the injected perturbation and the

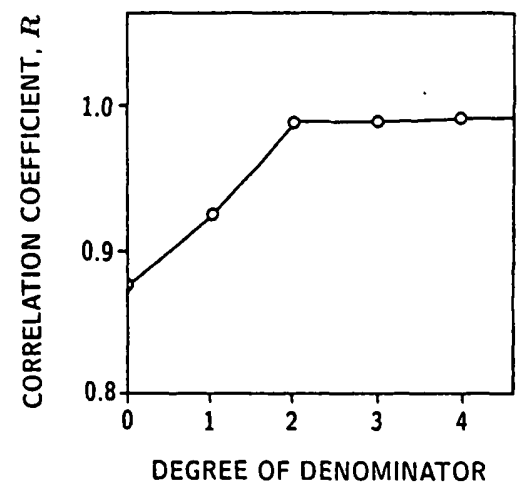

FIG. 8. Improvement of the correlation coefficient as the denominator order is increased. 
TABLE I. POLES AND ZEROES FOR DIFFERENT FITTING ORDERS (s-plane)

\begin{tabular}{lll}
\hline$(\mathrm{m}, \mathrm{n})$ & Poles $\left(\mathrm{s}^{-1}\right)$ & Zeroes $\left(\mathrm{s}^{-1}\right)$ \\
\hline$(0,1)$ & -391 & \\
$(1,2)$ & $-129 \pm j 589$ & 1839 \\
$(2,3)$ & $-130 \pm j 597$ & 1757 \\
& -3100 & -3441 \\
$(3,4)$ & $-154 \pm j 614$ & $126 \pm j 1430$ \\
& -403 & -326 \\
& -5397 & \\
\hline
\end{tabular}

plasma position. The transfer function between the applied voltage and the shell current will therefore have the same poles (the roots of the given characteristic equation), but it will have its own numerator.

The zeroes and poles together define the structure of the amplitude and phase plots of $\mathrm{H}^{*}(\mathrm{j} \omega)$, the estimated transfer function. Table I summarizes the fitted poles and zeroes obtained when these different orders were fitted. From $(p, q)=(1,2)$ onwards, the dominant poles $\left(-130 \mathrm{~s}^{-1} \pm j 600 \mathrm{~s}^{-1}\right)$ hardly move. The zeroes are not significant for our experimental conditions in which the perturbation is injected after the PD controller. When the order increases from $(1,2)$ to $(2,3)$, the new pole and the new zero $\left(-3100 \mathrm{~s}^{-1}\right.$ and $\left.-3441 \mathrm{~s}^{-1}\right)$ are very close, indicating that they almost factorize. This signifies that the fitting procedure was not able to use the extra degrees of freedom significantly, and that the new pole and the new zero taken together yielded a ratio close to unity, in which case the actual value of this zero and pole pair has almost no effect on the function $\mathrm{H}^{*}(\mathrm{~s})$. From $(p, q)=(2,3)$ onwards, there is no identifiable pole which might be taken into consideration for the feedback optimization. As the number of free coefficients increases, the modelled $\mathrm{H}^{*}(\mathrm{~s})$ will be adjusted to fit the available data. Unless a pole appears and remains insensitive to the presence of subsequent poles and zeroes fitted, we cannot consider it to be significant, and certainly cannot usefully apply it in a model in which the feedback controller could be optimized.

The correlation coefficient did not improve from second to fifth order and there were no new identifiable poles. Nonetheless, the amplitude and phase plots were slightly modified towards the higher frequencies (Fig. 9). These plots were obtained by substituting $s \rightarrow j \omega$ in the inverse bilinear transformation and evaluating the amplitude and the phase of $\mathrm{H}^{*}(\mathrm{j} \omega)$ for the frequency range plotted. The approximation of the physical system by the dominant second-order model $\mathrm{H}^{*}(\mathrm{~s})$ provides an excellent representation of the dominant feature of the closed-loop transfer function, which will determine the optimized controller settings.

The difference at higher frequencies is due to the fact that the poles added to the second-order transfer function are high frequency ones (Table I). These poles are statistically badly estimated, however, and this difference is not significant.

\subsection{Discussion of the required model properties}

Having established that the experimentally observed behaviour of the vertical position control loop is that of a low-order system, we re-examine the determining equations (4) through (7) proposed in Ref. [1]. Equation (4), which defines the instantaneous force balance, can clearly not be simplified any further. The second equation, that of the first antisymmetric vessel current mode, must obviously be retained since its time constant is a large part of the dynamical control problem. The third equation is of first order because of the term $R_{a}$, the coil resistance, and the derivative gain $G_{v}$ which we have added with the PD controller. The characteristic polynomial of these three determining equations is quadratic, i.e. it is the order found experimentally to be dominant.

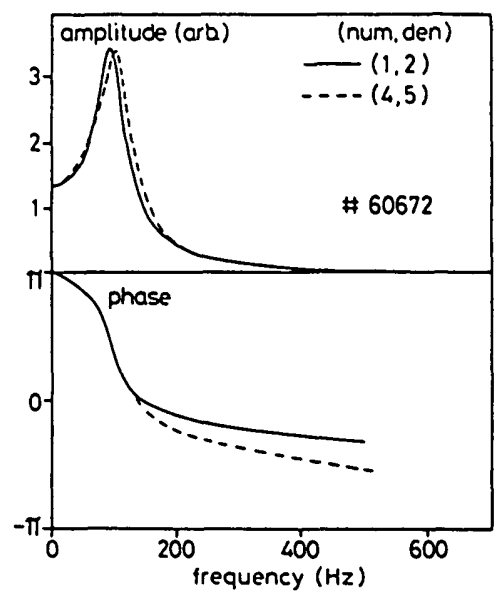

FIG. 9. Amplitude and phase plots of the modelled transfer function for second-and fifi-order denominators. 
This observation is extremely important. It implies that, in order to model the dominant vertical control features, we cannot require the presence of an additional dynamical equation, i.e. one which contains differing powers of s. Put in a different way, if we had started with a set of four equations, including a new fourth dynamical equation, we would not even have been able to identify the resulting model behaviour with the response observed on those particular tokamak discharges unless the resultant additional pole had only an effect at higher frequencies than those accessible with the experimental data.

This does not mean that Eqs (4)-(7) must be the exact equations defining the vertical movement. We are entitled to add a further equation, defining another coil current, for example, provided that this coil has no significant resistance, in which case its equation is essentially factorizable by s. This coil could also provide a radial field, modifying the first equation as well. Such an addition would not increase the order of the system or the possibility of providing a stable closed loop. The model would have the correct dynamical structure, but would not have perfectly correct coefficients. In the case of DIII-D with its many poloidal coils, those coils which are not part of the vertical feedback loop are nonetheless controlled by other shaping feedback loops with purely proportional gain. On our time-scale, these coils will appear to satisfy the criterion of zero resistance $\left(\gamma_{\mathrm{a}} \approx 0\right)$, and their presence does not raise the order of the vertical control loop. If we were to add all these additional coils to Eqs (4)-(7), we would find that we could perform a model reduction on the resulting set of equations, and return to equations similar in structure to those of Eqs (4)-(7). The coefficients would be modified because of the additional mutual inductances and their derivatives with respect to the plasma vertical position.

\section{VARIATION OF THE CONTROLLER GAINS AND THE DECAY INDEX}

In Section 3 we have seen that the coupled system of the vertical movement plus the vessel and active coil currents behaves predominantly as a second-order dynamical system. We expect that the effect of varying the controller gains, Eq. (7), will be similar to that predicted in Ref. [1], summarized in Fig. 1. We also expect that the system will become less stable as the decay index is increased. In this section we explore the results of experiments with such variations. First, we inspect the behaviour qualitatively; then we make use of the system identification method of Section 3 to extract the characteristic polynomial from the step response, and compare the real and imaginary parts of the poles with the model predictions.

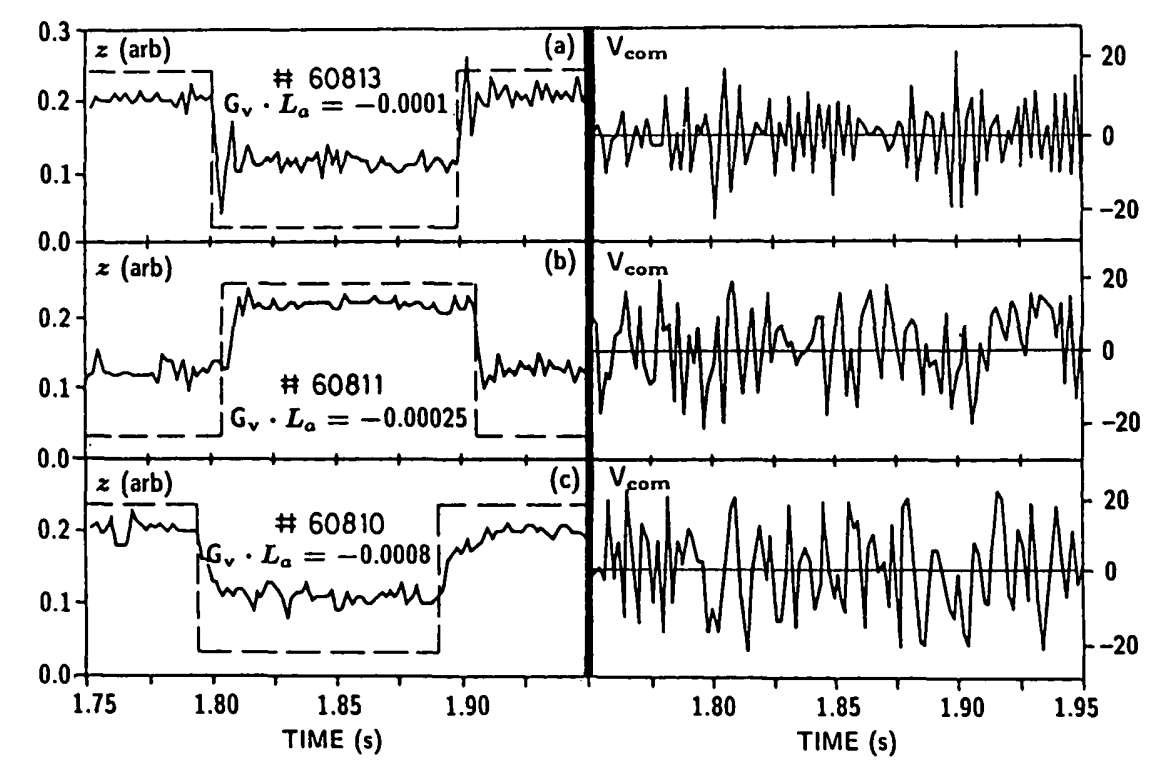

FIG. 10. Variation of the square wave response $z$ as the derivative gain is varied. $G_{v} \cdot L_{\mathrm{n}}=(a)-0.0001$, (b) $-0.00025,(c)-0.0008, G_{z}=-0.22$. The amplifier demand signal $\left(V_{c o m}\right)$ is also shown. 


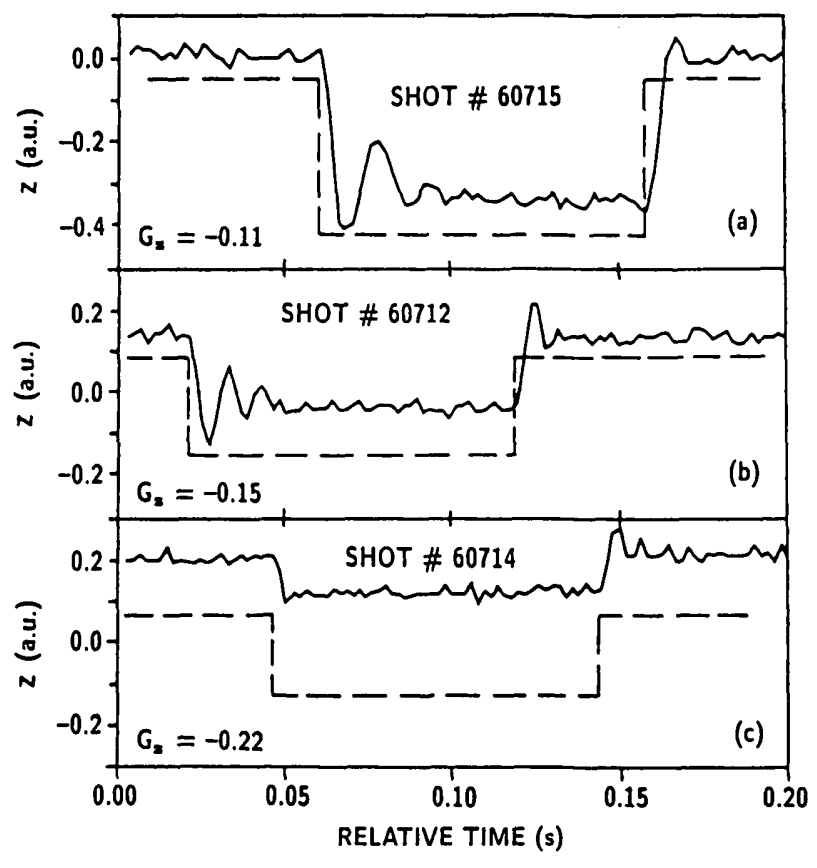

FIG. 11. Variation of the square wave response $z$ as the proportional gain is varied. $G_{z}=(a)-0.11,(b)-0.15$, (c) -0.22 , $G_{\mathrm{v}} \cdot L_{\mathrm{a}}=-0.00004$. The step response and voltage demand signals are shown.

\subsection{Varying the controller gains}

Using only the $F 7$ control coils in the feedback loop, we systematically varied $G_{z}$ and $G_{v}$. At a fixed value of $G_{z}=-0.15$, and with varying values of the derivative gain, $G_{v} \cdot L_{a}=-0.0008,-0.00025,-0.0001$ $\left(\mathrm{L}_{\mathrm{a}}=0.053 \mathrm{H}\right)$, we obtained the square-wave response results shown in Fig. 10, for a fixed value of the decay index, $n \approx 1.0$. As $-G_{v}$ is increased, the damping of the response is increased, as expected. The lowest damping, $G_{v} \cdot L_{a}=-0.0001$, led to an oscillatory response at the leading and trailing edge, Fig. 10(a). With $G_{v} \cdot L_{a}=-0.00025$, the response was close to critically damped, Fig. 10(b). Increasing the damping further, to $G_{v} \cdot L_{a}=-0.0008$, gave an excessively sluggish response, Fig. 10(c).

The damping of the response, obtained by increasing $-G_{v}$, also leads to an increase in the RMS fluctuation level of the amplifier demand signals, but not in the size of the response to the steps (Fig. 10). As $-G_{v} \cdot L_{a}$ increased from 0.0001 to 0.00025 and to 0.0008 , the RMS value of the demand signal increased from 8.9 to 10.1 to 12.1. The response of the leading and trailing edge is seen in the demand signal of Fig. 10(a), but not in Fig. 10(c). In the absence of noise on the position signal, we would expect a reduction in the demand signal as $-G_{v}$ is increased, as shown by the reduced presence of a response to the step. As long as the amplifier is not saturated, the presence of an increased signal due to $G_{v} \cdot s \cdot$ noise(s) is not detrimental to the control loop. However, the phase-amplitude relation of the controller output (Fig. 3(b)) requires that the bandwidth be extended beyond the characteristic frequency of the instability. Because of the noise in the position signal, the reduction of the demand signal with increased $-G_{v}$ is not seen. These data indicate how crucial the choice of the roll-off frequency will be when the controller is optimized.

Still using only the F7 coils, the proportional gain was then varied at a fixed value of the derivative gain, $\mathrm{G}_{\mathrm{v}} \cdot \mathrm{L}_{\mathrm{a}}=-0.00004$. The decay index remained at $\mathrm{n}=-1.0$. In Fig. 11(a), $\mathrm{G}_{\mathrm{z}}=-0.11$, the amplitude of the step is largest, and the oscillation frequency is lowest; the damping of the oscillation is also slowest. In Fig. 11(b), $G_{z}=-0.15$, the amplitude of the step is reduced, the oscillation frequency is higher and the damping is faster. Finally, in Fig. 11(c), $G_{z}=-0.22$, the oscillation is not visible in the noise, the response is rapid and the step amplitude is smallest. These observations agree with the predictions of Fig. 1, i.e. as $-G_{z}$ is increased, both $\gamma$ and $\omega$ increase as well.

\subsection{Effect of varying the decay index}

As the vertical field decay index becomes more negative, the results of Ref. [1] predict an increase in the more dangerous root of the transfer function, and subsequent loss of control at a certain decay index. For $\mathrm{n}<-\mathrm{n}_{\mathrm{a}}$, there is no stabilized solution without derivative gain, and for $n<-n_{c}$, there is no stabilized solution at all. Before this study, the loss of vertical control had always occurred in DIII-D at a decay index of $n \approx-0.95$, well above the value of $-n_{c} \approx-1.35$ which was calculated for the discharges studied. Using the control based on the F6 and F7 coils, we would calculate that $n_{a}$ should have a value of 0.65 , whereas the decay index achieved without a derivative gain was the value -0.95 mentioned above. We believe that this discrepancy results from the action of the equilibrium shape control, which tends to correct the vertical position through the change in flux at the controlling flux loops near the 18 coils when the plasma shifts position. In this way, the equilibrium control allows these 18 coils to act in a concerted effort, which is similar to adding a lumped second shell.

Figure 12 shows the square-wave response as the decay index is ramped from $n \approx-0.8$ to $n \approx-1.1$, with the controller gains set at $G_{2}=-0.22, G_{v} \cdot L_{a}=-0.0001$. 

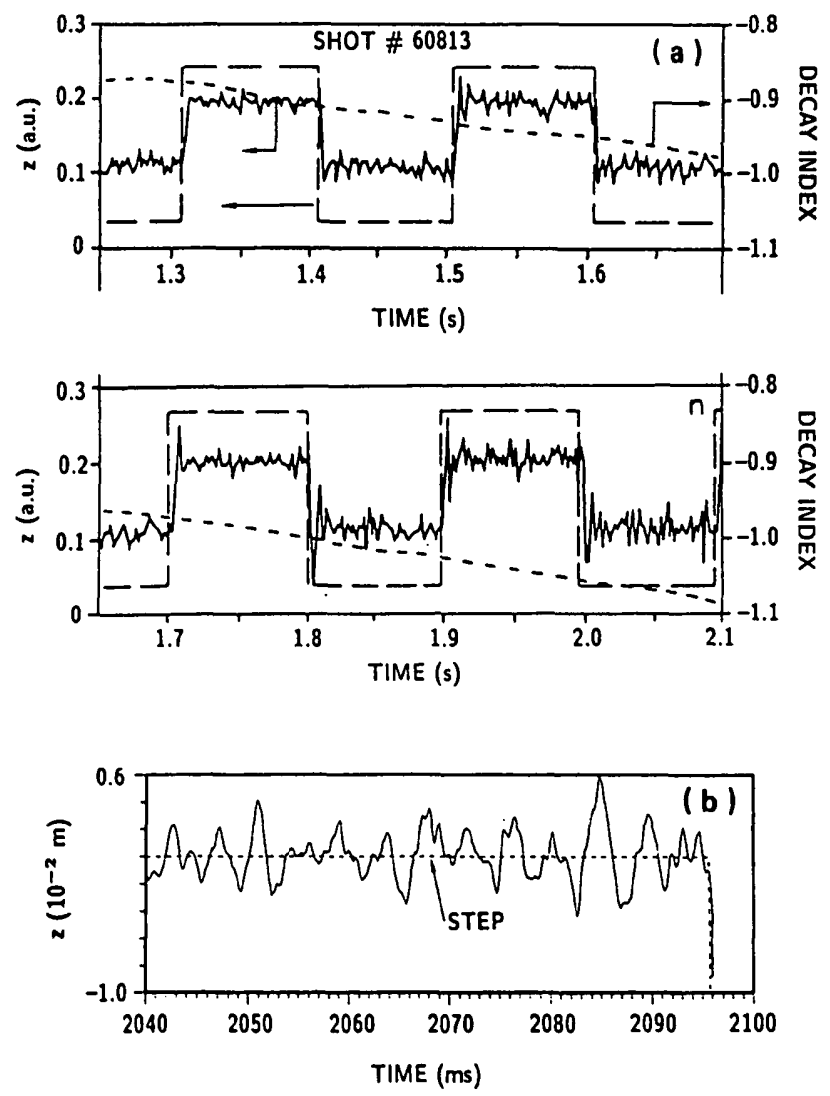

FIG. 12. (a) Evolution of the square wave response raw data as the vertical field decay index (dotted curve) increases during one discharge; $G_{z}=-0.15, G_{\mathrm{v}} \cdot L_{\mathrm{a}}=-0.0001$.

(b) Expanded time-scale for the last step. The disruption begins promptly at the step at $2.096 \mathrm{~s}$.

The response at the start of the ramp shows very little overshoot, being almost critically damped at $t \approx 1.3 \mathrm{~s}$. As the decay index decreases, the response starts to overshoot $(t \approx 1.5 \mathrm{~s})$, and by $t \approx 1.7 \mathrm{~s}$ the response is clearly oscillatory. The oscillations increase until, at $\mathrm{t} \approx 2.1 \mathrm{~s}$, the oscillation becomes unstable and $\mathrm{a}$ disruption ensues. In this discharge we have seen the predicted characteristic behaviour, namely that of the low-order system whose stability boundaries are moving as the decay index varies (Ref. [1], Fig. 9).

The discharge in Fig. 12 ended in a disruption of vertical control. Provided the proportional gain is adequate to keep the transfer function roots to the left of the line B-B of Fig. 1, a control disruption is easily identified by the control system, having necessarily passed through an oscillatory phase before becoming unstable. If the decay index evolves slowly enough with respect to the period of the square-wave steps, this natural response must be detected experimentally at a leading or trailing edge. The signal remains oscillatory after the step at $\mathrm{t}=1.996 \mathrm{~s}$, and then the plasma disrupts immediately upon the next step at $\mathrm{t}=2.096 \mathrm{~s}$. This is seen more easily at $\mathrm{t} \approx 2.1 \mathrm{~s}$ in Fig. 12(b), which shows the same signal digitized at a faster rate $(50 \mathrm{kHz})$. The higher frequency component distinguishable is the chopper frequency.

Disruptions which occur on a much faster timescale, and which are not correlated with the squarewave excitation, will not, in general, be avoidable by optimizing that particular control input which was stimulated by the square wave. This does not mean, however, that they are not controllable using other control loops, i.e. using either different detection coils, or poloidal field coils, or both.

Since the $G_{z}, G_{v}$ settings determine the closed-loop poles, we expect the operational range, i.e. the decay index at which the real part of the most dangerous root goes to zero, to vary with the controller gains. In fact, the discharges used in Fig. 10 show just such behaviour. In each case, we measured the maximum value of the decay index achieved just before the disruption (Fig. 13). The decay index achieved increased significantly after the addition of a significant derivative gain, from $\mathrm{n}=-0.96$ to $\mathrm{n}=-1.18$. Between $G_{v} \cdot L_{a}=-0.00025$ and $G_{v} \cdot L_{a}=-0.0008$, little improvement was found, as predicted by Fig. 20 of Ref. [1], calculated for a different value of $G_{2}$. The value of $n=-1.15$ is the limiting value calculated in Ref. [1] for vertical control by the F7 coils, in remarkable agreement with the experimental result. In each case, we measured the minimum value of the decay index for vertical control by the F7 coils, which was well above the critical value for these discharges $(\approx-1.35)$.

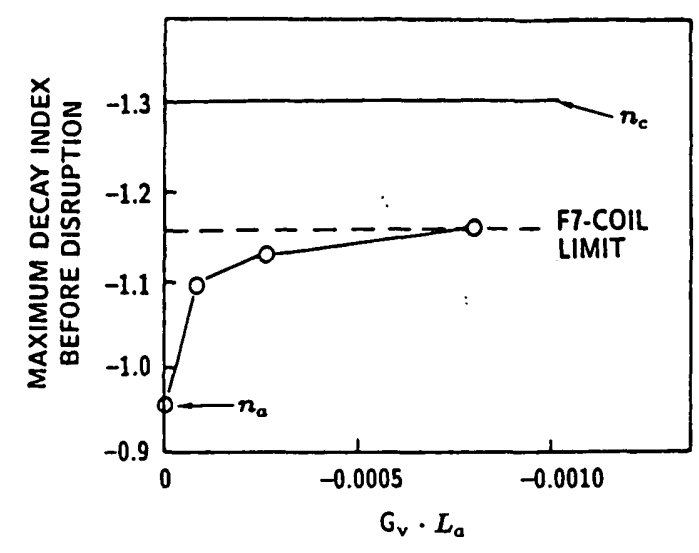

FIG. 13. Decay index achieved before distuption, as the F7 controller gains were varied. 


\subsection{Tracking the closed-loop poles}

The qualitative agreement with the model is extremely encouraging, and we looked for a more detailed agreement. For this purpose, we took the three discharges of Fig. 10 which provide decay index ramps for three different values of the velocity gain $G_{v}$. The data were analysed assuming a second-order denominator, and the two poles of the transfer function were estimated for each edge of the square-wave stimulation. The signal-to-noise ratio was poorer than before, since we could not box-car average over several periods, as had been done for the stationary conditions of Fig. 7. The $1 \mathrm{~ms}$ sampling period was a little too long. The results shown have been derived from the soft X-ray asymmetry factor, Eq. (9), although the magnetic measurement of $z(t)$ gave similar results, but with a greater uncertainty that was due to the longer sampling interval $(2 \mathrm{~ms})$.

Figure 14 shows the imaginary part $(\omega)$ and the real part $(\gamma)$ of the fitted closed-loop response poles. The

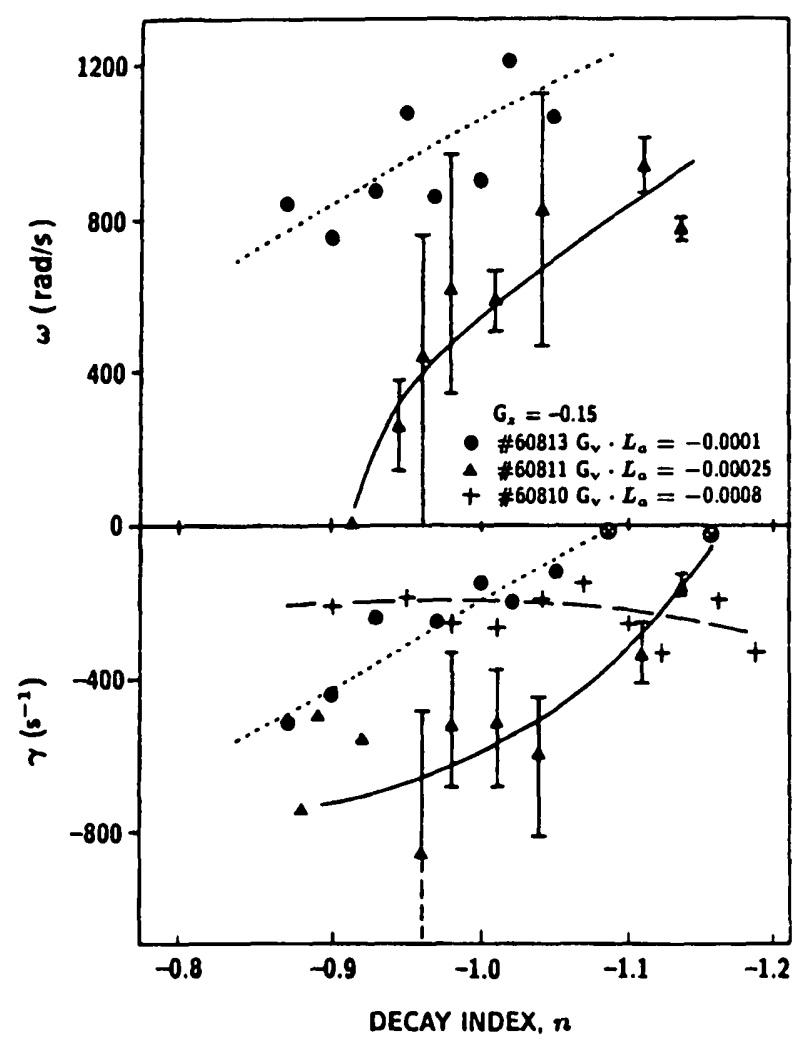

FIG. 14. Variation of the poles of the closed-loop response $H_{C L}(s)$ as the decay index was ramped, measured for three different velocity gains $(s=\gamma+j \omega)$.

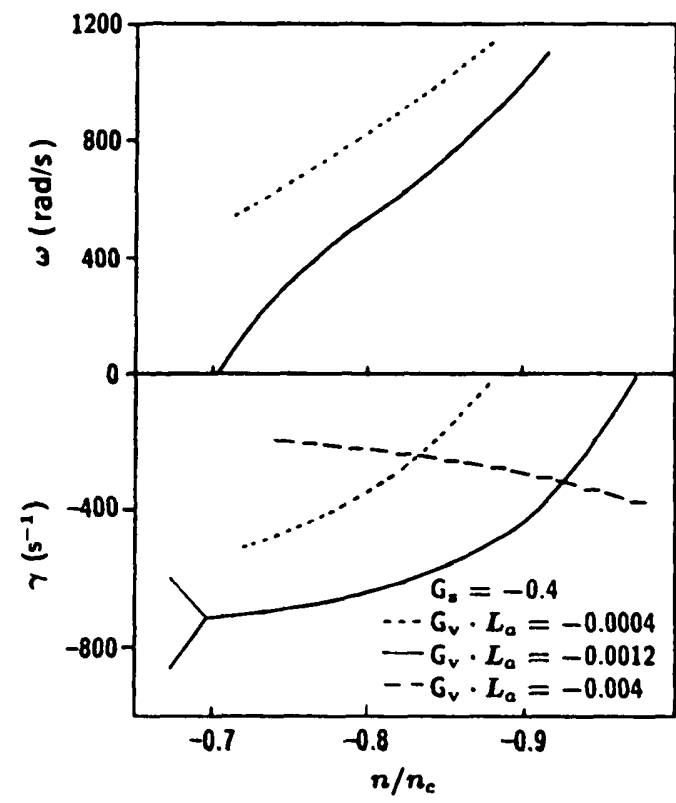

FIG. 15. Variation of the poles of the closed-loop response $H_{C L}(s)$, as a function of the decay index, calculated by the model $(s=\gamma+j \omega)$.

dotted line with the solid circles, $G_{v} \cdot L_{a}=-0.0001$, shows relatively high frequency oscillations. The growth rate is $\gamma \approx-500 \mathrm{~s}^{-1}$ at the start of the ramp and tends towards $\gamma \approx 0$ at $\mathrm{n}=-1.08$, at which point the plasma disrupted (open crossed circle). Increasing the velocity gain to $G_{v} \cdot L_{a}=-0.00025$ (solid line, solid triangles) gave an overdamped response at the start of the ramp $(\omega=0)$ and an oscillatory response above $n$ $\approx-0.92$. The oscillation frequency remained lower than in the previous case. The growth rate was more negative than before, and the $\gamma \approx 0$ disruption occurred at a higher value, $n \approx-1.15$. Increasing the velocity gain further to $G_{v} \cdot L_{a}=-0.0008$ produced a damped response for all decay indices $(\omega=0)$ and a growth rate $\gamma$ which was less negative than before and which increased negatively with the decay index ramp.

We derived the same curves from the model equations (4) through (7), maintaining the same ratio between the velocity and the proportional gains, and the same ratio between the different values of velocity gain. To obtain a reasonable quantitative match, we were forced to decrease the value of the shell time constant, increasing $R_{v}$ by $50 \%$. The absolute values of the gains were increased by a factor of four. The resulting curves, Fig. 15, are in astonishingly good agreement with the experimental data of Fig. 14. Bearing in mind that the shell time constant was not estimated with the diagnostic ports cut out, the change 


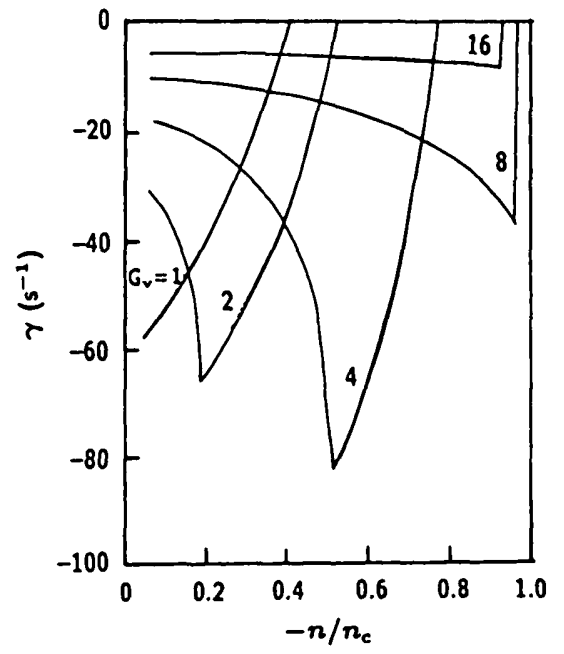

FIG. 16. The least stable root versus $-n / n_{c}$ for various $G_{\mathrm{v}}$ values using the $F 7$ coils. $G_{z}$ is constant. Gains are in arbitrary units.

of the time constant is not a cause for concern. The necessity of changing the gains is more disconcerting, although the fact that the presence of the remaining poloidal coils is not modelled correctly must have a significant effect here. We have already seen a change in $n_{a}$ from the predicted value, which indicates that the effective value of $\mathbf{M}_{\mathrm{ap}}^{\prime}$ must differ from our calculated value. In addition, there is an uncertainty in the transfer function of the choppers [4] which contributes to the overall circuit gain.

An important point to note in Figs 14 and 15 is that the disruption with the largest value of $G_{v}$ appears to occur at $n>-n_{c}$ while $\gamma$ is negative. The $F 7$ coils are incapable of controlling a discharge up to the critical index, as seen in Ref. [1], Fig. 20, and explained in Ref. [1], Section 5.1.2. For particular coils sets (F7, F8, and F9) the structure of the operating space is modified somewhat from Fig. 1 in that the line A-A is below the line $C-D$. In that case, the stable operating space is further limited by an upper bound on $-G_{v}$. As $\mathrm{n}$ approaches $-\mathrm{n}_{\mathrm{c}}$, the line $\mathrm{A}-\mathrm{A}$ moves upwards while the line C-D remains stationary. Eventually, the stable space vanishes before reaching $-n_{c}$. We now consider such a system in the experimental space of $\gamma$ versus $n / n_{c}$. The dependence of $\gamma$ on $n / n_{c}$ is shown in Fig. 16 for various values of derivative gain. Note that at high $G_{v}$ the stability is increasing just before the instability. Further increases in $G_{v}$ decrease the achievable range of $n$. This behaviour is characteristic of the use of coils which interact too strongly with the stabilizing shell currents. The F2 coils do not exhibit this behaviour, and the system stability is always reduced as $n$ approaches $-n_{c}$.
The trend of the poles as the decay index, the proportional gain and the derivative gain were varied is clearly of the form predicted by the model. The transfer function extracted from the data is in reasonable agreement with the calculated transfer function, but only if the values of two of the eight parameters which enter the model equations (4) through (7) are adjusted.

\section{HYBRID CONTROL OF THE VERTICAL MOVEMENT}

The vertical control model derived in Ref. [1] led us to the conclusion that the use of one poloidal coil pair is not optimal, and that the use of two selected coil pairs, one pair inboard and one pair outboard, would provide better vertical control. To a first approximation, the outboard coils give good positional rigidity by providing a large radial field per unit current, whereas they are very effectively shielded by the vessel image currents, making them ineffective for the production of the low amplitude variations in radial field at the high frequency necessary for vertical stabilization. The inboard coils provide a smaller radial field, which is inadequate for positional rigidity, whereas they are shielded by a vessel current distribution that has a much faster decay rate. This concept is referred to as hybrid control of the vertical movement.

We do not simply add the vertical control signal to all the coil pairs, since for many of the coils such a signal is actually destabilizing at high $n / n_{c}$ (see Ref. [1], Table II). The coil currents will induce vessel currents in opposition to those produced by the plasma motion, counteracting the vessel effectiveness on a fast timescale $\left(\omega>R_{v} / L_{v}\right)$. If we wish to improve the control of a coil pair, its net field, including that produced by its interaction with the vessel, must oppose the plasma motion.

The hybrid control was tested on similar discharges; for comparison, all discharges were driven to an axisymmetric disruption. Figure 17 is a plot of the decay index achieved before disruption, versus the derivative gain on the F7 coils. Our baseline for comparison with the simple control is the dashed line, which describes the discharges with only the F7 coils active (Fig. 13). Large values of feedback gain were used for the inboard coils, chosen to produce corrections of the order of the output range of the amplifiers. Adding only a large derivative feedback $\mathrm{G}_{\mathrm{vF}}$ to the F2 coils, marked by a crossed circle, a value of $\mathrm{n}=-1.18$ was achieved at low $\mathrm{G}_{\mathrm{v}} \cdot \mathrm{L}_{\mathrm{a}}(-0.001)$; this 


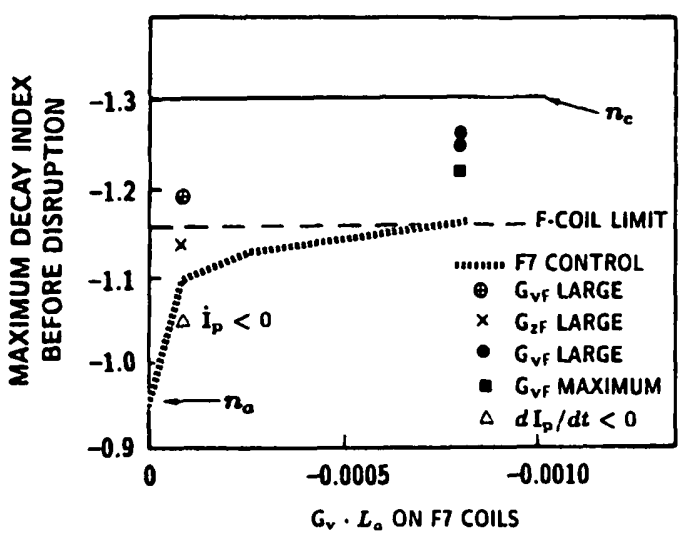

FIG. 17. Increase in the achievable decay index using the hybrid control. The data points of Fig. 13 are reproduced by the dashed line.

value exceeds the $\mathrm{F} 7$ coil optimum of $\mathrm{n}=-1.16$. Even adding only positional gain $G_{2 F}$ to the $F 2$ coils (marked by a cross) increased the operational range. Since increasing $G_{z}$ on the $F 7$ coils alone did not change the maximum achieved, this simple observation illustrates the difference in dynamics between inboard and outboard coil control. Our model simulations of this system also show that proportional gain on the F2 coils in conjunction with proportional gain on the F7 coils is stabilizing. These calculations indicate that this could not be an attractive option since the required power supply bandwidth would be higher than that for a system using derivative gain.

When the outboard coil velocity gain $G_{v} \cdot L_{a}$ was increased to -0.0008 , the additional velocity feedback on the $F 2$ coils $\left(G_{\mathrm{vF}}\right)$ produced a further significant increase in the decay index. These discharges are shown in Fig. 17 by the solid circles and the solid square. The maximum decay index was not obtained with the maximum velocity gain, although the small sample of discharges available cannot confirm the presence of a general optimum value of $G_{\mathrm{vF}}$, particularly since these high gains caused some saturation of the F2 coil drive signal. In shot No. 60809 we reached 92\% of the calculated maximum decay index for the measured plasma parameters.

The discharge marked by a triangle in Fig. 17 achieved a lower decay index, attributable to a decreasing plasma current following an accidental loss of control of the Ohmic primary circuit. Such a reduction in the decay index can be attributed to the direct dependence of the critical decay index on the plasma current ramp rate (Ref. [1], Section 2.3).
These data show that the predictions of a simple vertical control model led us to significant improvements in the achieved vertical field decay index, by providing a simple and clear picture of the controlled system. Such an improvement was obtained with a small amount of experimental data, contrary to the habitual trial and error optimization.

The data obtained with the hybrid vertical position control have not been subjected to such a dynamic analysis. Since we introduce a second poloidal coil pair with both proportional and derivative gain, the system model order will have increased by one. The proportional gain and coil resistance being small, there will be an extra pole with a small value. In addition, if we model the interaction with the vessel, we have to include the dominant mode $(\ell=6)$ interacting with the new coil pair. This mode would introduce a pole with a characteristic frequency well beyond the available data sampling rate since the $\ell=6$ eigenmode growth rate is about six times that of the dominant eigenmode. The characteristic knee that is seen when hybrid control is used would then not necessarily have moved, illustrating a common difficulty with the system analysis used.

The time history of shot No. 60809 is shown in Fig. 18. The shaping control system is programmed to provide a steadily increasing decay index once the plasma current has achieved a flat top. At $t=2.8 \mathrm{~s}$ the plasma disrupts, having exceeded $n / n_{c}=-0.92$. In the calculation of $n_{c}$ we have used the approximation

$\mathrm{L}_{\mathrm{ext}}=\mu_{0} \mathrm{X}_{0}\left[\ln \left(8 \mathrm{X}_{0} / \overline{\mathrm{a}}\right)-2\right]$
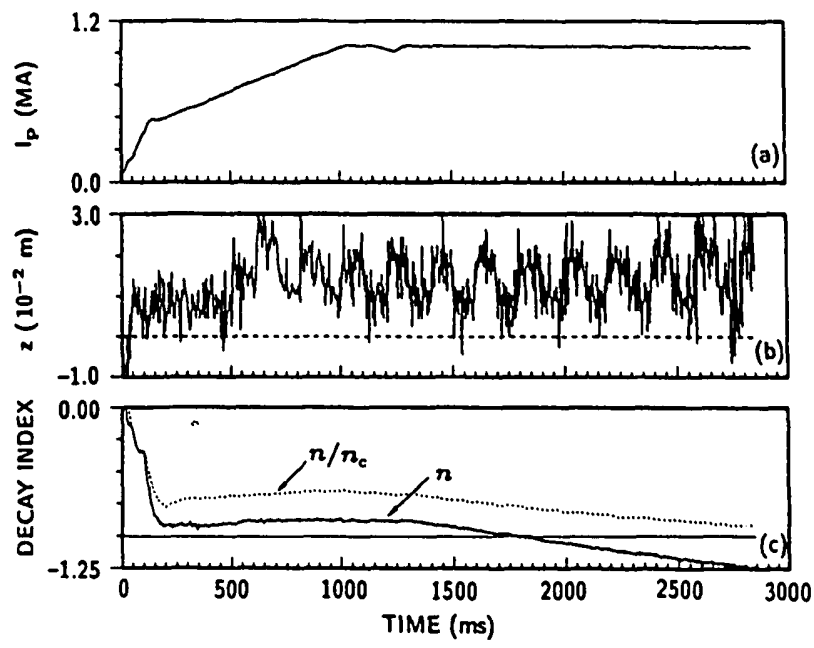

FIG. 18. Time evolution of shot No. 60809, which reached $92 \%$ of the ideal stability limit: (a) plasma current, (b) plasma vertical position, (c) $n / n_{c}$ and $n$. 


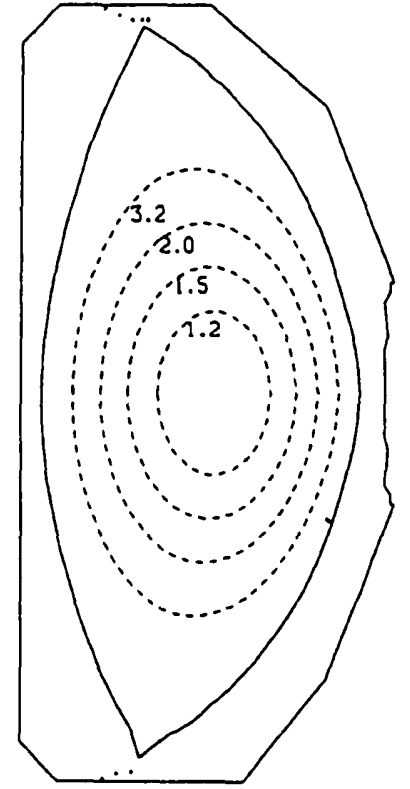

FIG. 19. Shot No. 60809, equilibrium approximately $0.1 \mathrm{~s}$ before disruption, as calculated from the experimental data. The parameters are: $I_{p}=1.0 \mathrm{MA}, B_{t}=2.0 \mathrm{~T}, X=1.68 \mathrm{~m}$, $a=0.59 m, q_{95}=5.6, \beta_{p}=0.37, \ell_{i}=1.5, \kappa=2.19$ and $\delta=0.37$.

\section{HIGHER ELONGATED PLASMAS}

Having established the improved performance of the hybrid control system compared with the previous control system, we attempted to shape higher elongation plasmas. One change, however, was made to the control circuit with respect to that discussed above. For the control circuit used, high gains are required, particularly in the case of the derivative gain. At the same time, the $\mathrm{z}$ measurement had a considerable noise component. This combination resulted in a large high-frequency demand on the power supplies and a tendency to saturate some of the intermediate circuitry. In an attempt to reduce these saturation effects, we applied the higher frequency control signal not only to the F2 coils but also to the F3 coils. (In Ref. [1] it was noted that the F3 coils are second only to the F2 coils in stabilizing the vertical plasma motion.) Similarly, the lower frequency drive was applied to the F6 coils as well as to the F7 coils. This effectively increased the overall derivative gain.

Now our strategy was twofold. The first consideration was to optimize the plasma shape. Since, to lowest order, a hexapole field is not vertically destabilizing, we decided to make high triangularity
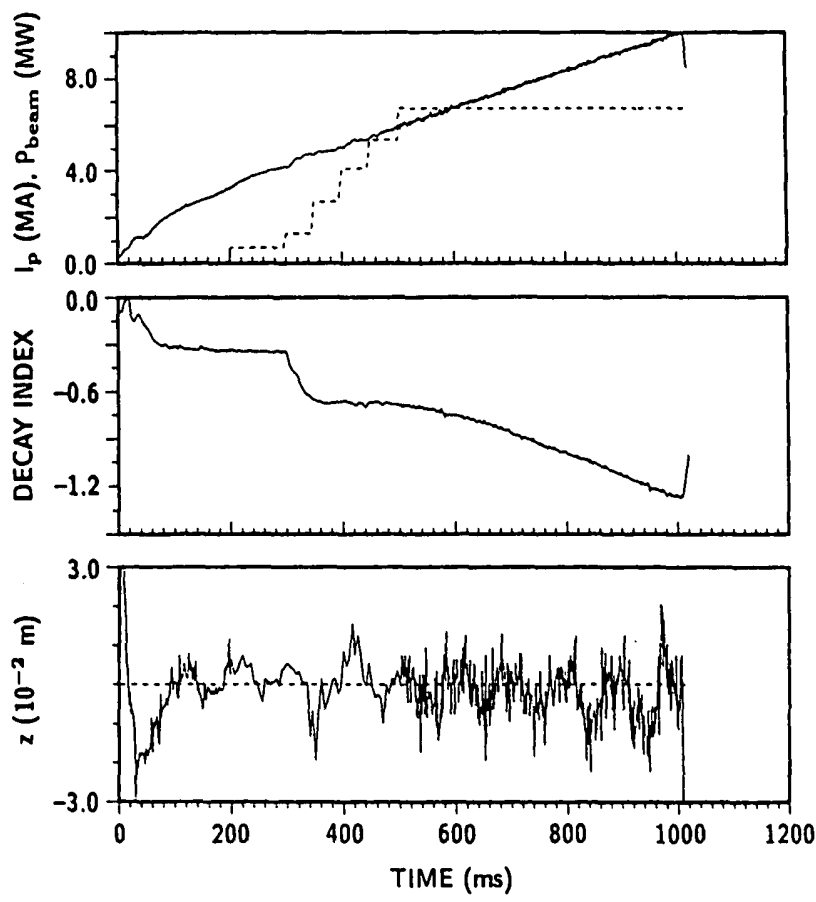

FIG. 20. Time evolution of shot No. 62458, which reached $\kappa=2.5$ before a vertical disruption: (a) plasma current and neutral beam power, (b) decay index, and (c) plasma vertical position. 


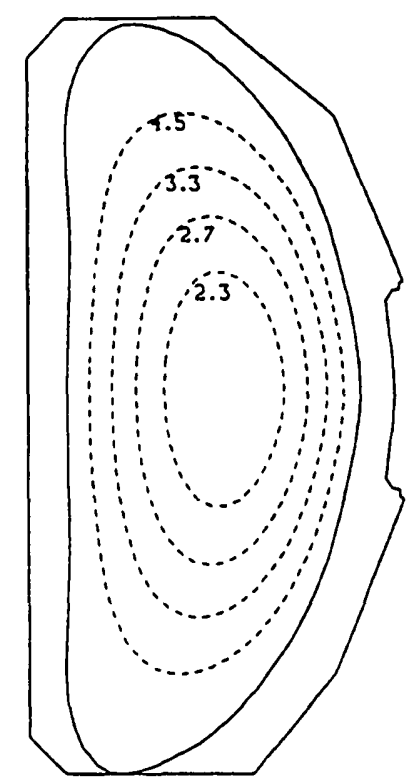

FIG. 21. Shot No. 63458, equilibrium just before disruption, as calculated from the experimental data. The parameters are: $I_{p}=1.0 \mathrm{MA}, B_{t}=1.7 \mathrm{~T}, X=1.69, a=0.54 \mathrm{~m}, q_{95}=7.7$, $\beta_{p}=0.51, \ell_{i}=0.94, k=2.50$ and $\delta=0.55$.

$(\delta \approx 0.75)$ plasmas. Secondly, we tried to reduce the internal inductance $\ell_{i}$. It is important to note that the relationship between the achievable elongation and the current profile is included in our simple filament model. Lowering $\ell_{i}$ both increases the critical index through a change in $\Gamma$ and reduces the quadrupole field (decay index) required to obtain any particular value of $k$.

So far, our best attempt has been an elongation $\kappa$ of 2.5. In this case, an $\ell_{i}$ value of 0.94 was reached with a combination of an $I_{p}$ ramp and intense neutral beam heating (Fig. 20). This plasma disrupted vertically, immediately after the equilibrium shown in Fig. 21. We were, however, able to sustain plasmas with $\kappa \approx 2.45$ for $500 \mathrm{~ms}$ during the plasma current flat top at $l_{i} \approx 1.04$. The $k=2.5$ plasma disrupted, with a value of $n / n_{c}=-0.85$, although there is some uncertainty which is due to possible contributions to the field curvature from the additional vessel current induced by the significant $I_{p}$ ramp.

\section{DISCUSSION}

The experimental behaviour of the DIII-D vertical control system is dominated by second-order dynamics. This agrees with the predictions of a simple model of the vertical control problem [1]. Varying the proportional and derivative gains of the controller gave results that are in agreement with this model. Good agreement at lower elongation $(\kappa-1.7)$ and with an iron core had been found previously in JFT-2M [10]. When the vertical field decay index was varied, the experimentally measured closed-loop system response poles varied in a way that is well described by the model calculations. The qualitative effects of the controller gain settings are in good agreement with the model. The quantitative agreement is also good, considering the uncertainties due to the presence of shaping coils that were not taken into consideration. This simple model therefore provides a means of systematically optimizing the control loop.

The concept of hybrid control of the vertical movement has been implemented and tested, leading to significant improvements in the achievable decay index.

The observed improvement achieved by the use of inboard coils, at least indirectly, confirms the supposition in Ref. [1] that the poloidal distribution of the vessel current is of prime importance in counteracting the problem of vessel shielding and that such currents flow predominantly on the outboard side of the vessel. The correct prediction of the apparently benign increase in stability, followed by disruption before $n=-n_{c}$ (Fig. 14 at high $G_{v}$ ), is strong confirmation of the importance of the vessel current distribution in the vertical control problem. It should be noted that with all the coils available in DIII-D, our model predicts that very few coils will provide adequate vertical stability (Ref. [1], Table II).

We have shown that periodical monitoring of the closed-loop performance using a square-wave pulse train makes it possible to identify the disruptions due to loss of control loop stability. The evolution of the control loop poles occurs on a time-scale on which a crude form of closed-loop adaptive control can be envisaged. The plasma response to an external stimulus allows us to determine whether the particular stimulated coils are useful or not for improving the plasma control.

It is encouraging to find that the plasma behaviour is dominated by a small number of poles, so that a simple controller may be used on highly elongated plasmas. The quest for the optimal set of control coils and detection coils can be undertaken experimentally with these systematic techniques.

In subsequent experiments we attempted to obtain an even higher elongation in a double-null configuration. This configuration provides the highest triangularity and permits $\mathrm{H}$-mode operation, which is also the easiest way to lower $f_{i}$. In these plasmas we found that we could no longer sustain $n / n_{c} \approx-1$. We saw a sys- 
tematic departure from this limit as the current profile was broadened, and at $f_{i} \approx 1$ we were only able to operate at approximately $60 \%$ of this ideal MHD limit, i.e. it was no longer possible to stabilize plasmas having much lower rigid-body growth rates than in the cases with higher $\ell_{i}$. Calculations with the PSTAB multi-filament model show an identical trend, and thus we conclude that this problem is not due to our singlefilament calculation of the rigid-body ideal limit. The plasma elongation was limited to 2.5 or less by this behaviour. These new vertical disruptions were not triggered by the response to a step, and we saw no oscillatory phase just before the disruption, as observed in shot No. 60809. Preliminary analysis with the GATO code indicates that the plasma is destabilized by the coupling of an $\mathrm{m} / \mathrm{n}=3 / 0$ mode to the $\mathrm{m} / \mathrm{n}=1 / 0$ mode. There is also some experimental evidence that this motion, which is not included in a rigid-body model, is present. It is not yet clear whether improvements of the control system will solve this problem. However, these plasmas are seen to disrupt at values of $n / n_{c}$ in the $H$-mode phase which were successfully sustained during the Ohmic (higher $\ell_{i}$ ) phase of the same discharge. New experiments are planned to investigate this in more detail, improving the control system and explicitly studying the effect of triangularity on $\mathbf{n}=0$ stability. Such behaviour has been observed in the PBX tokamak for bean-shaped plasmas [11], and a similar behaviour has been predicted for D-shaped plasmas with high triangularity [12]. The conclusion to be drawn from these latest findings is that the hybrid control described in this paper may have taken us to the useful limit of simple $\mathrm{m} / \mathrm{n}=1 / 0$ vertical stability control. The more complex control of these new high performance plasmas will be an exciting challenge.

\section{ACKNOWLEDGEMENTS}

Two of the authors (E.A. Lazarus and J.B. Lister) would like to thank R. Stambaugh, T. Taylor and the
DIII-D physics staff for their hospitality during their stay at General Atomics. The authors are grateful for the constructive comments by $P$. Noll during the preparation of this paper.

The work described was partly funded by Euratom, the Ecole polytechnique fédérale de Lausanne and the Fonds national suisse de la recherche scientifique, and by the Office of Fusion Energy, United States Department of Energy, under Contract Nos DE-AC0389ER511114 and DE-AC05-84OR21400.

\section{REFERENCES}

[1] LAZARUS, E.A., LISTER, J.B., NIELSON, G.H., Nucl. Fusion 30 (1990) 111.

[2] LUXON, J., ANDERSON, P., BATTY, F., et al., in Plasma Physics and Controlled Nuclear Fusion Research 1986 (Proc. 11th Int. Conf. Kyoto, 1986), Vol. 1, IAEA, Vienna (1987) 159.

[3] HOFMANN, F., JARDIN, S.C., MARCUS, F.B., PEREZ, A., TURNBULL, A., in Fusion Technology 1986 (Proc. 14th Symp. Avignon, 1986), Vol. 1, Pergamon Press, Oxford (1987) 687.

[4] ROCK, P.J., WESLEY, J.C., in Fusion Engineering (Proc. 8th Symp. San Francisco, 1979), Vol. 3, IEEE, New York (1979) 1289.

[5] MORET, J.M., Thesis No. 758, Rep. LRP 358/88, Ecole polytechnique fédérale de Lausanne (1988).

[6] JOYE, B., LISTER, J.B., MORET, J.M., POCHELON, A. SIMM, C.W., Plasma Phys. Controll. Fusion 30 (1988) 743

[7] JUNG, L., System Identification Theory for the User, Prentice-Hall, Englewood Cliffs, NJ (1987).

[8] BERNARD, L.C., HELTON, F.J., MOORE, R.W., Comput. Phys. Commun. 24 (1981) 377.

[9] LEUER, J., Fusion Technol. 152 (1989) 489.

[10] MORI, M., SUZUKI, N., SHOJI, T., YANAGISAWA, I., TANI, T., MATSUZAKI, Y., Nucl. Fusion 27 (1987) 725.

[11] JARDIN, S.C., DELUCIA, M., OKABAYASHI, M., et al., Nucl. Fusion 27 (1987) 569.

[12] BERNARD, L.C., BERGER, D., GRUBER, R., TROYON, F., Nucl. Fusion 18 (1978) 1331.

(Manuscript received 8 March 1990

Final manuscript received 18 July 1990) 